\title{
Blue and Double-peaked Green Receptors Depend on Ommatidial Type in the Eye of the Japanese Yellow Swallowtail Papilio xuthus
}

\author{
Michiyo Kinoshita, Daisuke Kurihara, Aiko Tsutaya \\ and Kentaro Arikawa* \\ Graduate School of Integrated Science, Yokohama City University, \\ Yokohama 236-0027, Japan
}

\begin{abstract}
The compound eye of the butterfly Papilio xuthus is composed of three spectrally distinct types of ommatidia. We investigated the blue and double-peaked green receptors that are encountered distally in type I and III ommatidia, by means of intracellular recordings, in vivo fluorescence microscopy, and histology. The blue receptors are R1 and/or R2 photoreceptors; they contain the same mRNA encoding the opsin of the blue-absorbing visual pigment. However, here we found that the sensitivity in the UV wavelength region strongly depends on the ommatidial type; the blue receptors in type I ommatidia have a distinctly depressed UV sensitivity, which is attributed to lateral filtering in the fused rhabdom. In the main, fronto-ventral part of the eye, the R3 and R4 photoreceptors of all ommatidia contain the same set of two mRNAs encoding the opsins of greenabsorbing visual pigments, PxL1 and PxL2. The spectral sensitivities are double-peaked, but the UV sensitivity of the R3 and R4 photoreceptors in type I ommatidia appears to be reduced, similar to that of the co-localized blue receptors.
\end{abstract}

Key words: color vision, compound eye, insect, retina, photoreceptor

\section{INTRODUCTION}

The compound eyes of insects are composed of three distinct types of ommatidia (Briscoe et al., 2003; Stavenga et al., 2001; Wakakuwa et al., 2005; White et al., 2003). The ommatidia of butterflies typically contain nine photoreceptor cells, R1-9. The visual organelle of a photoreceptor is the rhabdomere, which is composed of a stack of photoreceptive microvilli, and the rhabdomeres of the nine photoreceptors together form a fused rhabdom. The rhabdom of the Japanese yellow swallowtail, Papilio xuthus, is a long, slender cylinder, where the distal two-thirds consists of the rhabdomeres of photoreceptors R1-4; they are therefore called the distal photoreceptors. The proximal one-third of the rhabdom consists of the rhabdomeres of photoreceptors R58, the proximal photoreceptors. R9, the basal photoreceptor, contributes a minor section to the rhabdom immediately distal to the basement membrane.

The three types of ommatidia of Papilio xuthus are histologically distinguishable by colored pigment clusters in the photoreceptors, lining the rhabdom over the major part of its length (Fig. 1). The pigment clusters are red-colored in type I and II ommatidia, and yellow-colored in type III. The distal part of the rhabdom of type II ommatidia additionally con-

\footnotetext{
* Corresponding author. Phone: +81-46-858-1560;

Fax : +81-46-858-1560;

E-mail: arikawa@soken.ac.jp
}

Present address: School of Advanced Sciences, The Graduate University for Advanced Studies, Hayama 240-0193, Japan doi:10.2108/zsj.23.199 tains a pigment that strongly fluoresces under ultraviolet (UV) excitation light (Fig. 1). Therefore, under a fluorescence microscope with UV epi-illumination, type II ommatidia of the Papilio eyes light up like stars in the night sky (Arikawa et al., 1999a).

The eyes of Papilio xuthus express five mRNAs, each encoding a unique visual pigment opsin. The absorbance spectra, as predicted from the visual pigments' primary structures, peaked in the UV (Papilio xuthus UV, PxUV), blue (PxB) (Kitamoto et al., 2000), or long-wavelength region of the spectrum (PxL1, PxL2, PxL3) (Kitamoto et al., 1998). Histological localization of these opsin mRNAs in the retina revealed a marked heterogeneity of the ommatidia. The probe that specifically hybridized to the mRNA of PxUV (the PxUV probe) labeled the ommatidia in three characteristic patterns, depending on the ommatidial type. In type I ommatidia, the probe labeled either the distal R1 or the R2 photoreceptor, whereas in type II it labeled both R1 and R2. No labeling was detected in type III ommatidia. The PxB probe labeled all $\mathrm{R} 1$ and $\mathrm{R} 2$ photoreceptors that were not labeled by the PxUV probe, or the labeling by the PxB probe was strictly complimentary to that by the PxUV probe (Kitamoto et al., 2000). The PxL1 probe labeled both R3 and R4 photoreceptors in all ommatidial types in the main, frontoventral half of the eye, but neither R3 nor R4 in the dorsal

\section{ABBREVIATIONS}

UV, ultraviolet; V, violet; B, blue; dG, double-peaked green; sG, single-peaked green; R, red; BB, broadband. 


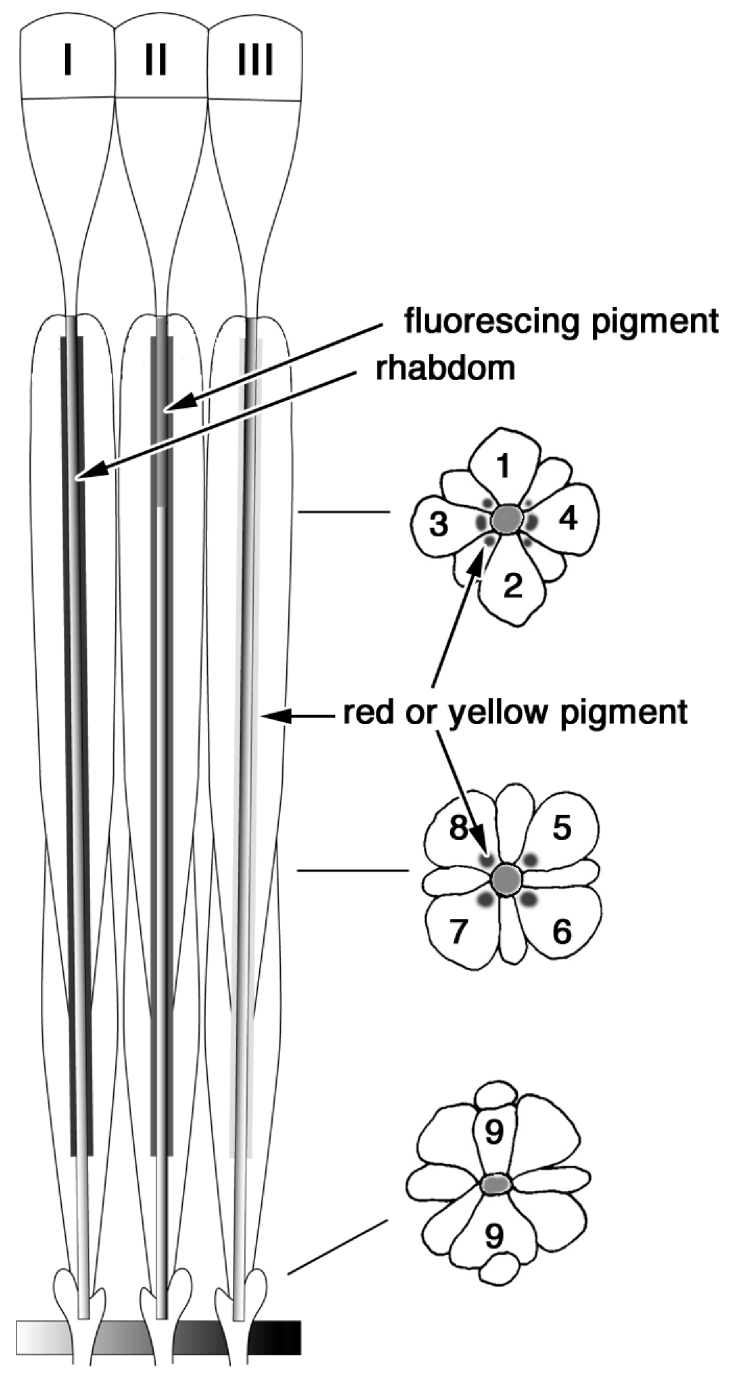

Fig. 1. Schematic drawing of three types of ommatidia, I-III, of Papilio xuthus, in longitudinal (left) and transverse views (right). 1-4, the distal photoreceptors R1-R4, which contribute their rhabdomeres to the fused rhabdom only in the distal two-thirds of the ommatidium; the cell soma of photoreceptors R3 and R4 contains pigment granules concentrated near the rhabdom. 5-8, the proximal photoreceptors R5-R8, which contribute their rhabdomeres to the fused rhabdom only in the proximal one-third of the ommatidium; the cell soma, which extends distally, contains pigment clusters near the rhabdom. 9, the basal photoreceptor R9, which contributes to its rhabdomere only in a short basal section of the rhabdom. The pigment clusters in photoreceptors R3-8 are red in type I and II ommatidia, and yellow in type III ommatidia. See also Table 1.

half. The PxL2 probe labeled R3 and R4 in all ommatidia, throughout the eye. In other words, R3 and R4 of the frontoventral ommatidia coexpress PxL1 and PxL2 mRNAs. The PxL2 probe also labeled the R5-8 photoreceptors of type II and III ommatidia, and the PxL3 probe labeled the R5-8 photoreceptors of both type I and II ommatidia. The R5-8 photoreceptors of type II ommatidia hence coexpress PxL2 and PxL3 mRNAs (Kitamoto et al., 1998). Finally, both PxL1 and PxL2 labeled the basal R9 photoreceptors in all ommatidial types (see Table I).

The Papilio eye is furnished with at least six classes of
Table 1. Three types of ommatidia in the eye of Papilio xuthus. R19, photoreceptors 1-9; UV, ultraviolet receptor; V, violet receptor; $B$, blue receptor; dG, double-peaked green receptor; sG, single-peaked green receptor; $\mathrm{R}$, red receptor; $\mathrm{BB}$, broad-band receptor. PxUV, opsin of Papilio xuthus UV-absorbing visual pigment; PxB, blueabsorbing visual pigment; PxL1-3, long wavelength-absorbing visual pigments 1,2 , and 3 . For details of the spectral sensitivities, $S(\lambda)$, see Arikawa (2003). For opsin structure and distribution, see Kitamoto et al. (1998, 2000). Asterisks: UV and B in type I ommatidia may be interchanged. The shaded (blue and double-peaked green) receptors of type I and III ommatidia are the topic of the present paper.

\begin{tabular}{|c|c|c|c|c|c|c|}
\hline \multirow{2}{*}{$\begin{array}{c}\text { Type of ommatidium } \\
\text { Pigmentation } \\
\text { Fluorescence } \\
\text { Photoreceptors }\end{array}$} & \multicolumn{2}{|r|}{$\begin{array}{c}\text { Type I } \\
\text { Red } \\
\text { No }\end{array}$} & \multicolumn{2}{|r|}{$\begin{array}{l}\text { Type II } \\
\text { Red } \\
\text { Yes }\end{array}$} & \multicolumn{2}{|r|}{$\begin{array}{l}\text { Type III } \\
\text { Yellow } \\
\text { No }\end{array}$} \\
\hline & $S(\lambda)$ & opsin & $S(\lambda)$ & opsin & $S(\lambda)$ & opsin \\
\hline $\mathrm{R} 1$ & $U^{*}$ & PxUV & V & PxUV & B & PxB \\
\hline $\mathrm{R} 2$ & $B^{*}$ & PxB & V & PxUV & B & PxB \\
\hline R3-4 & $d G$ & PxL1+PxL2 & $s G$ & $P x L 1+P x L 2$ & $d G$ & PxL1+PxL2 \\
\hline R5-8 & $\mathrm{R}$ & PxL3 & BB & $P x L 2+P x L 3$ & $d G$ & PxL2 \\
\hline R9 & $R / G ?$ & PxL1+PxL2 & $\mathrm{R} / \mathrm{G}$ ? & $P x L 1+P x L 2$ & $\mathrm{R} / \mathrm{G}$ ? & PxL1+PxL2 \\
\hline
\end{tabular}

spectral receptors, which are categorized as UV, violet, blue, green, red (Arikawa et al., 1987), and broad-band (Arikawa et al., 2003). We localized these spectral receptors in the ommatidia by intracellular recording and dye injection. In the distal tier, R1 and R2 appeared to be UV, violet, or blue receptors, whereas R3 and R4 were always found to be green sensitive (Bandai et al., 1992). The proximal R5-8 photoreceptors were of the green, red, or broadband classes (Arikawa et al., 2003). R9 basal photoreceptors were green or red-sensitive (Arikawa and Uchiyama, 1996).

We correlated the molecular biological data on the photoreceptors with their spectral sensitivities, and used optical modeling to deduce the visual pigment spectra from the measured sensitivity spectra. R1 and R2 labeled with the PxUV probe in type I ommatidia were thus proven to be UV receptors, whereas those in type II ommatidia were found to be violet $(\mathrm{V})$ receptors (Table 1). The spectral shift to the violet is caused by the fluorescing pigment in type II ommatidia, which acts as a UV-absorbing optical filter. The filter reduces the photoreceptors' sensitivity on the short wavelength side, and thus changes the PXUV-containing $\mathrm{R} 1$ and R2 cells into violet receptors (Arikawa et al., 1987). The green-sensitive R3 and R4 photoreceptors simultaneously express PxL1 and PxL2. The R3-4 photoreceptors in type I and III ommatidia have a sensitivity spectrum with a secondary peak in the UV, and they are therefore called double-peaked green (dG) receptors. The fluorescing, UVabsorbing filter causes the loss of the secondary sensitivity peak of the R3-4 receptors in type II ommatidia, and they are therefore called single-peaked green ( $\mathrm{sG}$ ) receptors (Arikawa et al., 1999a; Bandai et al., 1992). In the proximal tier, the R5-8 photoreceptors in type I ommatidia, which contain PxL3, are red receptors, which can be understood as resulting from the combined action of an orangeabsorbing visual pigment and a red-transmittant filter pigment (Arikawa et al., 1999b). The R5-8 photoreceptors in type III ommatidia, which contain PxL2, are double-peaked green receptors, due to a green-absorbing visual pigment 
and a yellow-transmittant filter pigment (Arikawa et al., 1999b). The R5-8 photoreceptors in type II ommatidia, which coexpress PxL2 and PxL3, have a broad sensitivity spectrum, stretching from $450 \mathrm{~nm}$ to $650 \mathrm{~nm}$, that results from the coexpression of green- and orange-absorbing visual pigments and a red-transmittant filter (Arikawa et al., 2003).

The R1 and R2 photoreceptors that express PxB were blue receptors (Table 1). Careful inspection of the spectral sensitivities of the blue receptors revealed that their spectra depended on ommatidial type. The R3-4 green receptors appeared to exhibit similar dependencies (Table 1). Here we report these findings and discuss the hypothesis that the spectral differences are caused by optical filtering within the fused rhabdom.

\section{MATERIALS AND METHODS}

\section{Animals}

Spring-form males of the Japanese yellow swallowtail butterfly Papilio xuthus were taken from a laboratory culture that was derived from eggs laid by females caught in the field around the campus of Yokohama City University. The hatched larvae were reared on fresh citrus leaves at $28^{\circ} \mathrm{C}$ under $8 \mathrm{~h}$ light: $16 \mathrm{~h}$ dark cycle. The pupae were stored at $4^{\circ} \mathrm{C}$ for at least 3 months and then allowed to emerge at $26^{\circ} \mathrm{C}$. Butterflies were used within 3 days after emergence.

\section{Electrophysiology}

Electrophysiological methods were as described elsewhere (Arikawa et al., 2003). Briefly, a glass microelectrode filled with $5 \%$ Lucifer yellow $\mathrm{CH}(\mathrm{LY})$ in $1 \mathrm{M} \mathrm{LiCl}_{2}$ was inserted into the distal tier of the retina through a hole made in the cornea. The optical axis of the penetrated photoreceptor was located by moving the position of a point light source of $1.6^{\circ}$, using a Cardan arm perimeter device, until dim white light flashes produced a maximal response. The spectral type of the penetrated photoreceptor was first determined by stimulating it with a series of monochromatic light from a $500 \mathrm{~W}$ Xenon arc lamp equipped with one of 22 narrow-band interference filters, with peak transmittance in the wavelength range $300 \mathrm{~nm}$ to $700 \mathrm{~nm}$. The maximum quantum flux of each monochromatic stimulus at the corneal surface was adjusted to $5.0 \times 10^{11}$ photons $\mathrm{cm}^{-2}$ $\mathrm{s}^{-1}$. We then recorded the response-light intensity (V-log I) function at the peak wavelength. When the maximal response amplitude exceeded $30 \mathrm{mV}$, we injected LY into the photoreceptors by applying a hyperpolarizing current of 2-6 nA to the electrode for approximately $5 \mathrm{~min}$. The ommatidium containing the LY-injected receptor was identified with an epifluorescence microscope (BX-60; Olympus, Tokyo, Japan) applying violet excitation (dichroic cube UMNBV). We subsequently switched the excitation light to UV (dichroic cube U-MNBV) to assess whether the ommatidium of the stained photoreceptor contained the UV-fluorescing pigment, that is, was a type II ommatidium.

\section{Histology}

The compound eyes containing LY-injected receptors were processed for light-microscopic histology. The eyes were isolated and fixed in $4 \%$ paraformaldehyde in $0.1 \mathrm{M}$ sodium cacodylate

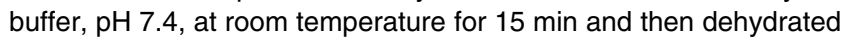
with an acetone series and embedded in Quetol 812 resin. Sections (10-14 $\mu \mathrm{m}$ thick) cut with a rotary microtome were first inspected with the epifluorescence microscope applying violet excitation to retrieve the LY-injected photoreceptor, and subsequently observed with transmitted white light to identify the color of the pigment clusters in the ommatidium of the stained cell. Only when a single photoreceptor was marked in the preparation did we gather the electrophysiological data.

\section{RESULTS}

We analyzed in this study a total of 28 photoreceptors, 19 blue and 9 double-peaked green receptors. All of them were identified histologically, as shown in the examples of Figs. 2 and 3.

\section{Blue receptors}

We encountered 19 photoreceptors with peak sensitivity at $460 \mathrm{~nm}$, which were accordingly categorized as blue (B) receptors. Fig. $2 \mathrm{~A}$ shows the spectral sensitivity of a blue receptor. It has a rather sharp profile, with a prominent peak at $460 \mathrm{~nm}$. The ommatidium containing this particular photoreceptor appeared to be non-fluorescing (Fig. 2B, C) and red pigmented (Fig. 2E) and thus is of type I (see also Table 1). The photoreceptor was identified as R1 (Fig. 2D, E; see Fig. 1). Fig. 3 is an example of a blue receptor in a non-fluorescing (Fig. 3B, C) and yellow-pigmented (Fig. 3E) ommatidium, which is therefore of type III. The photoreceptor was identified as R2. The spectrum of this particular photoreceptor shows high sensitivity in the UV wavelength region, but the UV sensitivity varies greatly from preparation to preparation (see Fig. 4). The cause of the variation has not yet been determined.

Nine of the measured blue receptors were R1 or R2 photoreceptors in type I ommatidia, whereas ten were R1 or R2 in type III ommatidia. Figure 4 shows the average spectral sensitivities of the two sets of blue receptors, together with the (normalized) absorption spectrum of a visual pigment with peak wavelength at $460 \mathrm{~nm}$ (Govardovskii et al., 2000). It appears that the UV sensitivity of the blue receptors in type I ommatidia is strongly reduced compared to the visual pigment absorption spectrum. The spectral sensitivity of the blue receptors in type III ommatidia does not significantly differ from the visual pigment spectrum.

\section{Double-peaked green receptors}

We analyzed 9 double-peaked green (dG) receptors, four in type I ommatidia and five in type III ommatidia, which were identified as R3 or R4 photoreceptors (see Table 1). Fig. 5 presents the average spectral sensitivities of the two sets of receptors, together with the (normalized) absorption spectrum of a mixture of two visual pigments, R515 and R545 (Govardovskii et al., 2000), in the ratiot of 8:2 (for details, see Discussion). In addition to their main sensitivity band in the green wavelength range, all double-peaked green receptorshad a secondary sensitivity peak in the UV range. The UV sensitivity of the dG receptors in type III ommatidia appears to be significantly higher than that predicted from the green visual pigments.

\section{DISCUSSION}

\section{Lateral filtering}

The R1 and R2 photoreceptors of type I and type III ommatidia appeared to have spectral sensitivities peaking at about $460 \mathrm{~nm}$ (Fig. 4). The photoreceptors contain the same visual pigment (PxB; Kitamoto et al., 2000) that we conclude to be an R460 visual pigment, because the absorption spectrum of R460 matches reasonably well the sensitivity spectrum of the blue receptors in type III ommatidia (Fig. 4). The R460 spectrum, which is calculated with a visual pigment 
A
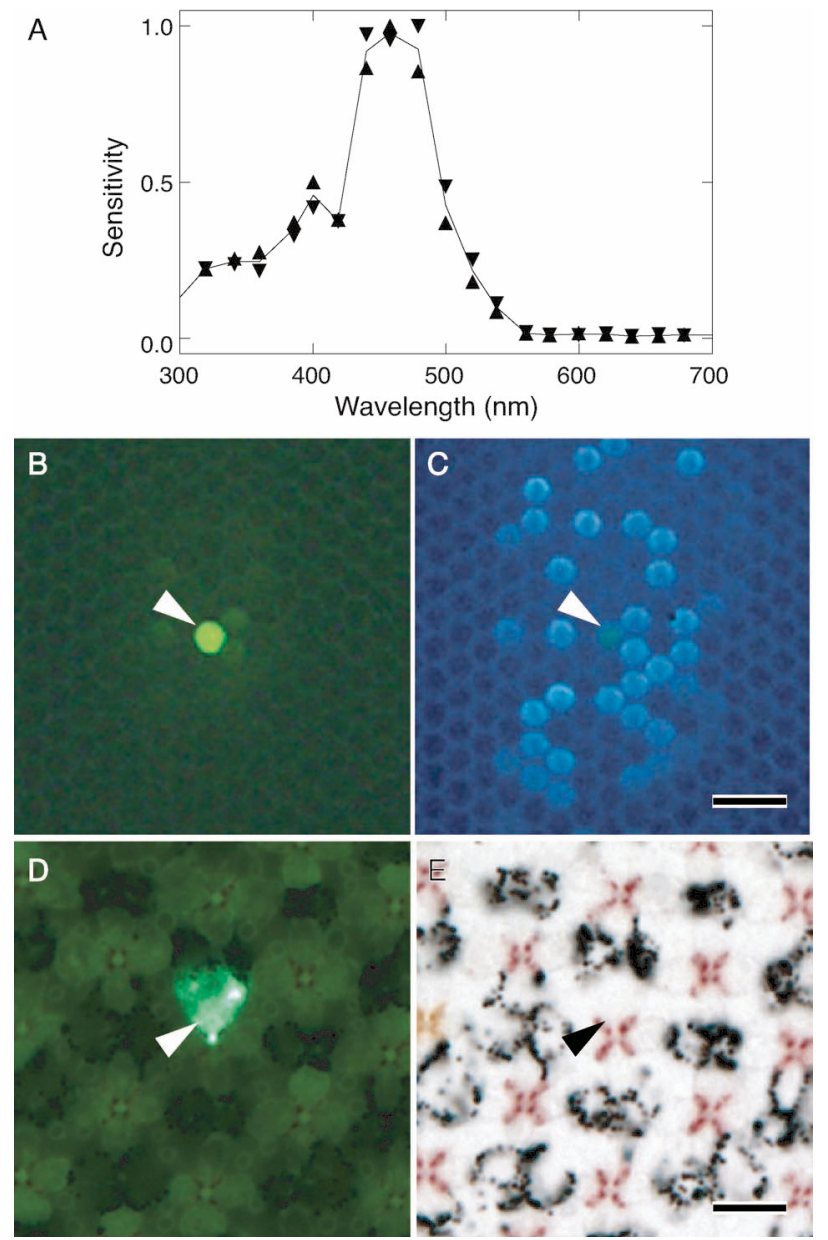

Fig. 2. Blue receptor in a type I ommatidium. (A) Spectral sensitivity determined by intracellular recording. Recording was made twice, first from shorter to longer wavelength $(\boldsymbol{\Delta})$ and then in reverse $(\boldsymbol{\nabla})$. Solid line indicates the average of two spectral runs. (B) Green fluorescence induced by violet epi-illumination reveals the ommatidium (arrowhead) containing the Lucifer yellow-injected photoreceptor. (C) Blue fluorescence induced by UV epi-illumination shows that the ommatidium of the recorded photoreceptor (arrowhead) is not of type II (see Table I). (D) Green fluorescence induced by violet epi-illumination of a transverse section of the eye proves that the photoreceptor labeled with Lucifer yellow is an R1 (arrowhead; see Fig. 1). (E) A transverse section observed with transmitted light, showing that the ommatidium containing the Lucifer yellow-injected R1 (arrowhead) is red-pigmented. Scale: $100 \mu \mathrm{m}$ (B,C), $10 \mu \mathrm{m}(\mathrm{D}, \mathrm{E})$.

template (Govardovskii et al., 2000), approximates the spectral sensitivity of the type I blue receptors in the blue region, but the spectra markedly differ in the UV wavelength region. This difference is probably an optical one, likely due to spectral filtering of the light propagating in the fused rhabdom.

Lateral filtering is a phenomenon that occurs in fused rhabdoms, where the photoreceptors' rhabdomeres are joined together into a single light-guiding rhabdom (Snyder et al., 1973). The rhabdomeres mutually act as spectral filters, especially when each rhabdomere contains different visual pigments. The rhabdoms of type I ommatidia contain in the distal tier a UV (R1 or R2), a blue (R2 or R1), and two double-peaked green receptors (R3 and $R 4)$, whereas in the
A
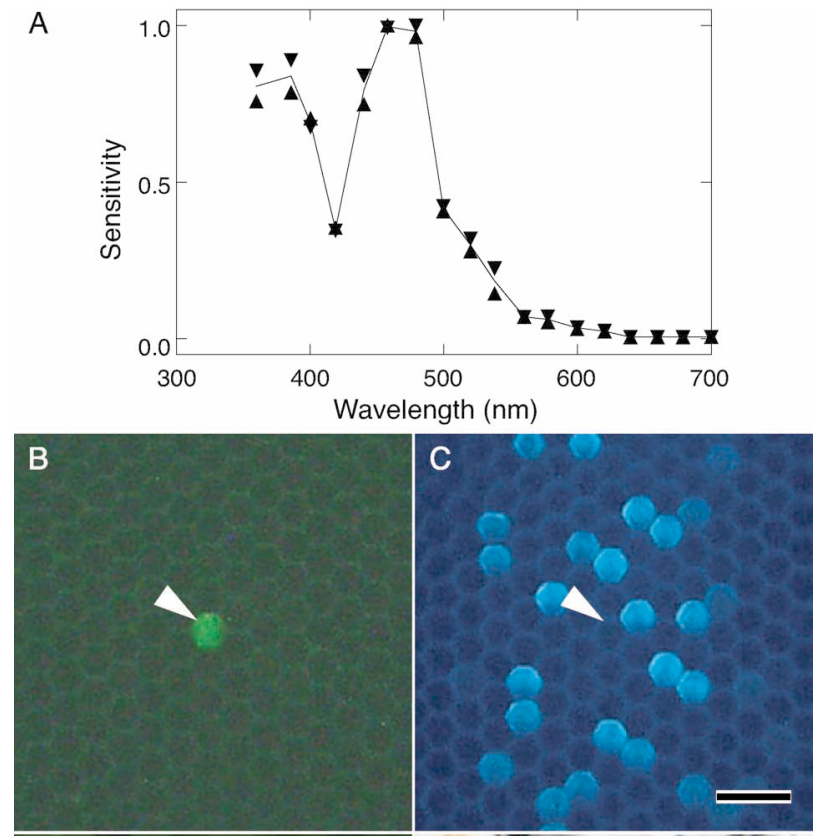

\section{D}

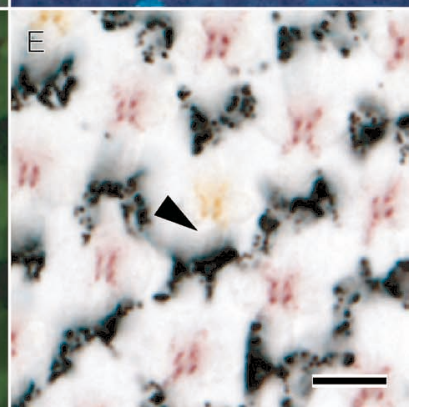

Fig. 3. Blue receptor in a type III ommatidium. (A) Spectral sensitivity determined by intracellular recording. Recording was made twice, first from shorter wavelength to longer wavelength $(\boldsymbol{\Delta})$ and then in reverse $(\boldsymbol{\nabla})$. Solid line indicates the average of two spectral runs. (B) Green fluorescence induced by violet epi-illumination reveals the ommatidium (arrowhead) containing the Lucifer yellowinjected photoreceptor. (C) Blue fluorescence induced by UV epiillumination shows that the ommatidium of the recorded photoreceptor (arrowhead) is not of type II. (D) Green fluorescence induced by violet epi-illumination of a transverse section of the eye proves that the photoreceptor labeled with Lucifer yellow is an R2 (arrowhead; see Fig. 1). (E) A transverse section observed with transmitted light, showing that the ommatidium containing the Lucifer yellow-injected $\mathrm{R} 2$ (arrowhead) is yellow-pigmented. Scale: $100 \mu \mathrm{m}$ (B,C), $10 \mu \mathrm{m}$ $(\mathrm{D}, \mathrm{E})$.

distal part of the type III rhabdoms there are two blue (R1 and R2) and two double-peaked green (R3 and R4) receptors. The rhabdomere of the UV receptors in the type I rhabdom thus acts as a UV-absorbing spectral filter for other members of the rhabdom, resulting in a reduced sensitivity of co-localized blue receptors at short wavelengths.

The R3-4 photoreceptors of type I ommatidia have a lower UV sensitivity than the R3-4 photoreceptors of type III ommatidia (Fig. 5). This difference might be attributed to the lateral filtering effect of UV-absorbing rhabdomeres in type I ommatidia, but we have to consider here that we performed our recordings in the main, fronto-ventral region of the eye. The R3 and R4 photoreceptors there coexpress two long- 


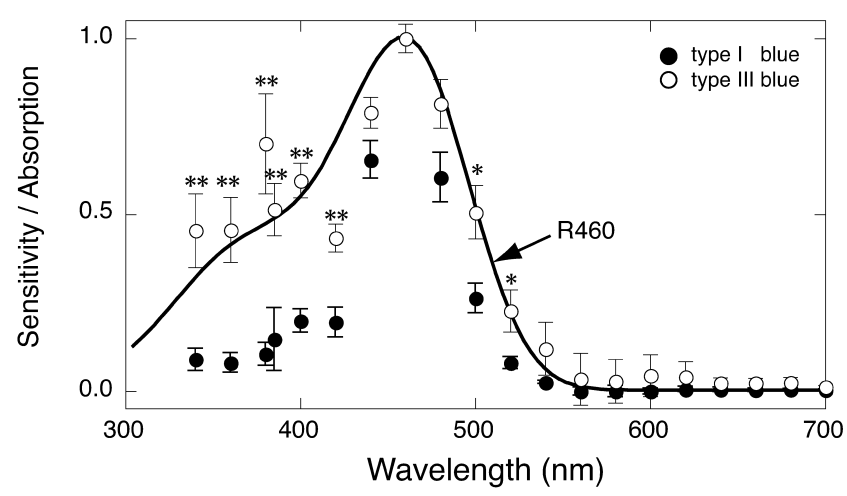

Fig. 4. Averaged spectral sensitivities of blue receptors in type I (,$n=9)$ and type III $(\bigcirc, n=10)$ ommatidia. The blue receptors in type I ommatidia have a strongly reduced sensitivity in the shortwavelength region of the spectrum, compared to those in type III ommatidia (** indicates $P<0.01 ; *$ indicates $\mathrm{P}<0.05$; $t$-test). Bars: standard errors.

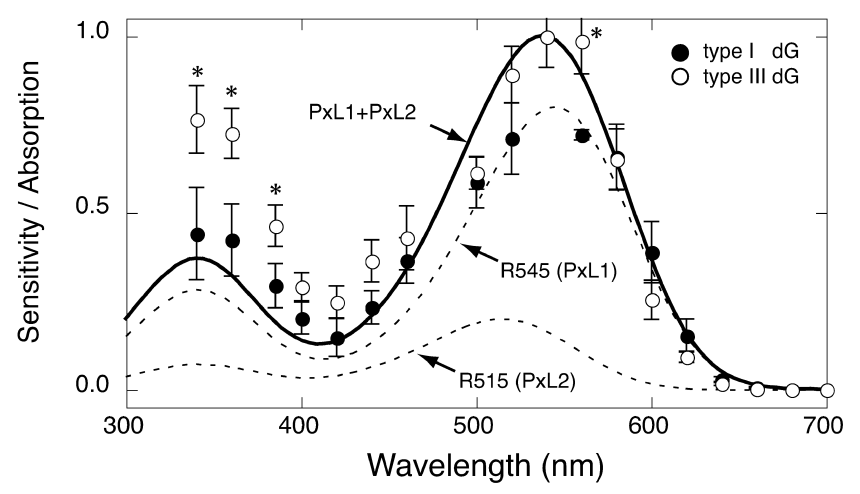

Fig. 5. Averaged spectral sensitivities of double-peaked green (dG) receptors in type I $(O, n=4)$ and type III $(\bigcirc, n=5)$ ommatidia. Type III dG receptors have a reduced sensitivity in the short-wavelength region of the spectrum $(* *$ indicates $P<0.01 ; *$ indicates $\mathrm{P}<0.05 ;$-test). Bars: standard errors.

wavelength absorbing visual pigments, PxL1 and PxL2 (Kitamoto et al., 2000). PxL2 has been assessed to be an R515 visual pigment with a peak absorption at $515 \mathrm{~nm}$, based on an analysis of the spectral sensitivity of R5-8 proximal green receptors in type III ommatidia (Arikawa et al., 1999b). The characteristics of PxL1 have not yet been determined. We hypothesized that PxL1 is an R545 visual pigment, and that the ratio of PxL1 and PxL2 in R3 and R4 photoreceptors is 8:2. We then obtained a summed absorption spectrum, again based on the template by Govardovskii (2000), that reasonably matches the spectral sensitivity of dG receptors of type I ommatidia (Fig. 5). If lateral filtering causes the sensitivity difference of type I and III dG receptors, the sensitivity spectrum of type III dG receptors should then be closer to the spectrum predicted from the visual pigment absorption. The type III dG receptors have a significantly higher sensitivity in the UV, however. The UV sensitivity may be somehow enhanced in the photoreceptors containing PxL1 and PxL2 visual pigments, but the mechanism is not known.

\section{Functional implications}

The eye of Papilio is a random mesh of three spectrally distinct types of ommatidia (Table 1). The random array of spectral receptors is probably crucial for color vision (Arikawa, 2003). Could the variations in the blue and double-peaked green receptors serve to enhance the ability of color discrimination? This may not be the case for the double-peaked green receptors, but it could be quite possibly so for blue receptors.

Several lines of evidence indicate that the R3 and R4 photoreceptors are not involved in color vision. First, the R3 and R4 of all ommatidia are maximally sensitive in the green, thus forming a complete hexagonal array of green receptors in the retina that is well-suited for achieving high spatial acuity, for instance for motion vision (Bandai et al., 1992; Kitamoto et al., 1998). The uniqueness of the R3-4 system is also clear from the organization of the first optic ganglion, the lamina. The axons of the R3 and R4 photoreceptors are large in diameter, have no branches (Takemura et al., 2005), and bear numerous synaptic specializations on the axon surface, with second order neurons in the lamina (Takemura and Arikawa, 2005). Such an organization should be advantageous for fast signal transmission, which is required for motion detection. Green receptors mediate motion detection in many insects, including butterflies (Hausen and Egelhaaf, 1989; Horridge et al., 1984; Kaiser and Liske, 1974). Therefore, the observed differences in the spectral sensitivities of R3 and R4 in the UV range will not be crucial for color vision.

The other classes of spectral receptors, including blue receptors, have thinner axons that bear either long or short axon collaterals and make extensive synaptic contacts with multiple photoreceptor classes. The neuronal network constructed by the inter-photoreceptor connections is ommatidial type-specific (Takemura and Arikawa, 2005), suggesting that the network is strongly related to spectral information processing. The two types of blue receptors, as well as the UV and violet receptors, contribute to the network, and therefore are most likely involved in spectral coding. Of course, there are green receptors that participate in the neuronal network, namely R5-8 of type III ommatidia (Table 1), but R3 and R4 do not participate (Takemura and Arikawa, 2005; Takemura et al., 2005).

\section{ACKNOWLEDGMENTS}

We thank Dr. Doekele Stavenga for critical reading of the manuscript. The work was supported by the Grants-in-Aid for Scientific Research from the JSPS to MK (17770062) and to KA (14204080), and a Grant from Yokohama Academic Foundation to MK.

\section{REFERENCES}

Arikawa K (2003) Spectral organization of the eye of a butterfly Papilio. J Comp Physiol A 189: 791-800

Arikawa K, Uchiyama H (1996) Red receptors dominate the proximal tier of the retina in the butterfly Papilio xuthus. J Comp Physiol A 178: 55-61

Arikawa K, Inokuma K, Eguchi E (1987) Pentachromatic visual system in a butterfly. Naturwissenschaften 74: 297-298

Arikawa K, Mizuno S, Scholten DGW, Kinoshita M, Seki T, Kitamoto J, Stavenga DG (1999a) An ultraviolet absorbing pigment causes a narrow-band violet receptor and a single-peaked 
green receptor in the eye of the butterfly Papilio. Vision Res 39: $1-8$

Arikawa K, Scholten DGW, Kinoshita M, Stavenga DG (1999b) Tuning of photoreceptor spectral sensitivities by red and yellow pigments in the butterfly Papilio xuthus. Zool Sci 16: 17-24

Arikawa K, Mizuno S, Kinoshita M, Stavenga DG (2003) Coexpression of two visual pigments in a photoreceptor causes an abnormally broad spectral sensitivity in the eye of a butterfly, Papilio xuthus. J Neurosci 23: 4527-4532

Bandai K, Arikawa K, Eguchi E (1992) Localization of spectral receptors in the ommatidium of butterfly compound eye determined by polarization sensitivity. J Comp Physiol A 171: 289297

Briscoe AD, Bernard GD, Szeto AS, Nagy LM, White RH (2003) Not all butterfly eyes are created equal: rhodopsin absorption spectra, molecular identification and localization of UV- blue- and green-sensitive rhodopsin encoding mRNA in the retina of Vanessa cardui. J Comp Neurol 458: 334-349

Govardovskii VI, Fyhrquist N, Reuter T, Kuzmin DG, Donner K (2000) In search of the visual pigment template. Vis Neurosci 17: $509-528$

Hausen K, Egelhaaf M (1989) Neural mechanisms of visual course control in insects. In "Facets of Vision" Ed by DG Stavenga, RC Hardie, Springer-Verlag, Berlin, pp 391-424

Horridge GA, Marcelja L, Jahnke R (1984) Color vision in butterflies 1. Single colour experiments. J Comp Physiol A 155: 529-542

Kaiser W, Liske E (1974) Die optomotorische Reaktionen von fixiert fliegenden Bienen bei Reizung mit Spektrallichtern. J Comp Physiol 89: 391-408
Kitamoto J, Sakamoto K, Ozaki K, Mishina Y, Arikawa K (1998) Two visual pigments in a single photoreceptor cell: Identification and histological localization of three mRNAs encoding visual pigment opsins in the retina of the butterfly Papilio xuthus. J Exp Biol 201: 1255-1261

Kitamoto J, Ozaki K, Arikawa K (2000) Ultraviolet and violet receptors express identical mRNA encoding an ultraviolet-absorbing opsin: Identification and histological localization of two mRNAs encoding short-wavelength-absorbing opsins in the retina of the butterfly Papilio xuthus. J Exp Biol 203: 2887-2894

Snyder AW, Menzel R, Laughlin SB (1973) Structure and function of the fused rhabdom. J Comp Physiol 87: 99-135

Stavenga DG, Kinoshita M, Yang EC, Arikawa K (2001) Retinal regionalization and heterogeneity of butterfly eyes. Naturwissenschaften 88: 477-481

Takemura S, Arikawa K (2005) Ommatidial type-specific inter-photoreceptor connections in the lamina of the swallowtail butterfly, Papilio xuthus. J Comp Neurol 494: 663-672

Takemura S, Kinoshita M, Arikawa K (2005) Photoreceptor projection reveals heterogeneity of lamina cartridges in the visual system of the Japanese yellow swallowtail butterfly, Papilio xuthus. J Comp Neurol 483: 341-350

Wakakuwa M, Kurasawa M, Giurfa M, Arikawa K (2005) Spectral heterogeneity of honeybee ommatidia. Naturwissenschaften 92: 464-467

White RH, Xu H, Munch T, Bennett RR, Grable EA (2003) The retina of Manduca sexta: rhodopsin-expression, the mosaic of green- blue- and UV-sensitive photoreceptors and regional specialization. J Exp Biol 206: 3337-3348

(Received October 31, 2005 / Accepted November 25, 2005) 